\title{
SYNTHESIS OF LAYERED SHELLS FROM A FINITE SET OF VISCOELASTIC MATERIALS
}

\author{
E. A. BONDAREV ${ }^{1}$, V. A. BUDUGAEVA ${ }^{1}$ and E. L. GUSEV ${ }^{1}$
}

(Received 15 July 1996; revised 11 April 1997)

\begin{abstract}
The method suggested earlier for solving the problems of optimal design from a limited set of elastic materials is generalized to a viscoelasticity case. The computational experiment for the problem of free oscillations of a spherical shell shows that characteristics of a viscoelastic layered structure may be improved due to peculiarities of wave propagation through the boundaries of layers made of different materials.
\end{abstract}

\section{Introduction}

The present state of the problem of optimal design of composite structures from a given set of materials with different restrictions on their behavior has been adequately described in the monographs $[2,4,5]$. In particular, these monographs present the method developed by the authors to solve the problems of synthesis of elastic layered cylindrical shells that provide necessary damping of oscillations under different external effects and with the restrictions on the mass or total thickness of a structure.

The problems of free oscillations hold a specific position among the problems of dynamics of elastic structures. This position is explained by the fact that free oscillation characteristics (fundamental frequencies and forms) fully determine the individual properties of a mechanical system; they are also of prime importance in analysing its forced oscillations. In this connection, the problems of structure synthesis from a finite set of materials, with different restrictions on free oscillation frequency, are of particular interest. The first and the only publication concerning this aspect is the paper by V. V. Alyokhin [1] where the problem of synthesis of the layered cylinder and sphere of minimum mass has been considered.

The wide application of polymeric materials in machinery makes it necessary to study the problems of optimal design of inhomogeneous structures having viscoelastic

\footnotetext{
'Institute of Physical-Technical Problems of the North, Octyabrskaya Str. 1, 677891 Yakutsk, Russia. (C) Australian Mathematical Society 1999, Serial-fee code 0334-2700/99
} 
properties. Therefore it is of great interest to analyse the peculiarities of the problems of optimal control of viscoelastic systems in relation to similar problems for elastic structures. Such analysis is also important because the damping characteristics of viscoelastic materials may be sufficient to make them preferable for the design of some structures. The problem is to determine whether viscoelasticity is a suppressing physical factor of reflection and refraction effects at the boundaries between different layers.

There are many publications concerning the solution of direct problems of estimating the characteristics of viscoelastic structures (see, for example, [6,9]). The most important scientific result obtained by the authors of the above papers lies in establishing the nonmonotonic dependence of dissipative characteristics of structures made of viscoelastic materials on their geometric and other parameters of structure inhomogeneity. This result can be used as the basis for stating the problem of synthesis of layered structures from viscoelastic materials with restrictions ensuring the realization of practically important structure requirements (minimum weight, maximum damping decrement, etc.).

\section{An illustrative example}

As an example, let us consider a multilayered spherical shell, where each layer is filled with a viscoelastic material, the mechanical properties of which depend on a layer number $n$. We then analyse the effect of its structure (viscoelastic parameters of materials, relative arrangement and thickness of layers), synthesized from a finite set of viscoelastic materials, on damping of free oscillations.

Problems of free oscillations belong to a class of problems where inertial terms fully determine material behavior. Therefore it is necessary that boundary conditions correspond to the work of all the external forces being zero. Masses must also be assumed to be zero.

With regard to the correspondence principle, the direct problem of free oscillations of a viscoelastic spherical shell can be solved in the same way as the corresponding problem of elasticity theory, with moduli replaced by the complex viscoelastic ones.

Free oscillations of a homogeneous sphere from a viscoelastic material are described by the following boundary value problem:

$$
\begin{gathered}
\frac{\partial \sigma_{r}}{\partial r}+2 \frac{\sigma_{r}-\sigma_{\varphi}}{r}=\rho \frac{\partial^{2} u}{\partial t^{2}}, \\
\sigma_{r}=(\bar{\lambda}+2 \bar{\mu}) \frac{\partial u}{\partial r}+2 \bar{\lambda} \frac{u}{r}, \\
\sigma_{\phi}=2(\bar{\lambda}+\bar{\mu}) \frac{u}{r}+\bar{\lambda} \frac{\partial u}{\partial r}, \quad R_{1}<r<R_{2},
\end{gathered}
$$




$$
\sigma_{r}\left(R_{1}\right)=\sigma_{r}\left(R_{2}\right)=0 .
$$

Here $R_{1}$ and $R_{2}$ are inner and outer radii of a spherical shell, $\bar{\lambda}$ and $\bar{\mu}$ re viscoelastic parameters of Lamé which can be written (as in [3]) as

$$
\left.\begin{array}{c}
\bar{\lambda}_{n}=\lambda_{n}\left[1-\Gamma_{\lambda n}^{c}\left(\omega_{R}\right)-i \Gamma_{\lambda n}^{s}\left(\omega_{R}\right)\right] \\
\bar{\mu}_{n}=\mu_{n}\left[1-\Gamma_{\mu n}^{c}\left(\omega_{R}\right)-i \Gamma_{\mu n}^{s}\left(\omega_{R}\right)\right]
\end{array}\right\},
$$

where $\lambda_{n}, \mu_{n}, R_{\lambda n}, R_{\mu n}$ are Lamé parameters and relaxation kernels of $n$-layer material (for a homogeneous sphere $n=1$ ) and $\omega_{R}$ is a real constant.

The solution of the problem (2.1)-(2.4) for $n=1$ can be presented in the form

$$
\begin{gathered}
u(r)=\left(C_{1}+C_{2}\right)\left\{-\frac{\kappa^{2}}{r} \cos (\kappa r)+\frac{\kappa}{r^{2}} \sin (\kappa r)\right\} \\
-i\left(C_{1}-C_{2}\right)\left\{\frac{\kappa}{r^{2}} \cos (\kappa r)+\frac{\kappa^{2}}{r} \sin (\kappa r)\right\} \quad \text { and } \\
\sigma_{r}(r)=\left(C_{1}+C_{2}\right)\left[\frac{4 \mu \kappa}{r} \cos (\kappa r)-\left(\frac{4 \mu}{r^{2}}-\kappa^{2}(\lambda+2 \mu)\right) \sin (\kappa r)\right] \frac{\kappa}{r} \\
+i\left(C_{1}-C_{2}\right)\left[\frac{4 \mu \kappa}{r} \sin (\kappa r)+\left(\frac{4 \mu}{r^{2}}-k^{2}(\lambda+2 \mu)\right) \cos (\kappa r)\right] \frac{\kappa}{r},
\end{gathered}
$$

where $\kappa^{2}=\rho \omega^{2} /(\lambda+2 \mu)$, the bar over $\lambda$ and $\mu$ being omitted.

In determining constants from boundary conditions (2.4), one can obtain the characteristic equation

$$
\begin{aligned}
\cos [ & \left.\kappa\left(R_{1}-R_{2}\right)\right]\left\{\frac{4 \mu \kappa}{R_{1}}\left(\frac{4 \mu}{R_{2}^{2}}-\rho \omega^{2}\right)-\frac{4 \mu \kappa}{R_{2}}\left(\frac{4 \mu}{R_{1}^{2}}-\rho \omega^{2}\right)\right\} \\
& +\sin \left[\kappa\left(R_{1}-R_{2}\right)\right]\left\{\frac{(4 \mu \kappa)^{2}}{R_{1} R_{2}}+\left(\frac{4 \mu}{R_{1}^{2}}-\rho \omega^{2}\right)\left(\frac{4 \mu}{R_{2}^{2}}-\rho \omega^{2}\right)\right\}=0 .
\end{aligned}
$$

In the case of elastic materials, the coefficients $\lambda$ and $\mu$ are real, which corresponds to the equalities $\bar{\lambda}=\lambda, \bar{\mu}=\mu$ in (2.5). The solution (2.9) may now be checked 
TABLE 1. Characteristics of elastic materials and corresponding frequencies of fundamental oscillations of a spherical shell.

\begin{tabular}{|c|c|c|c|c|}
\hline No. of the material & $\rho$ & $\lambda$ & $\mu$ & $\omega$ \\
\hline 1 & 1 & 6 & 6 & 7.04 \\
\hline 2 & 2 & 16 & 16 & 8.14 \\
\hline 3 & 4 & 34 & 34 & 8.39 \\
\hline
\end{tabular}

using results obtained by V. V. Alyokhin [1]. The values of parameters are given in Table 1. It is also assumed that $R_{1}=0.8$ and $R_{2}=1.0$. Natural frequency values for each material presented in Table 1 perfectly coincide with V. V. Alyokhin's results. While describing viscoelastic materials, volume strain has been considered to be pure elastic, that is, modulus of volume compressibility $K=\lambda+\frac{2}{3} \mu$ is a constant. To describe shearing strain, the Rzhanitsyn-Koltunov's relaxation kernel has been used [7] as the most common one in mechanics of polymers:

$$
R_{\mu}=A \exp (-\beta t) / t^{1-\alpha} .
$$

Solutions (2.7) and (2.8) can be easily generalized to a case of a multilayered sphere. Denote

$$
\begin{aligned}
& \Delta_{n}=\left(c_{n}\left(r_{n-1}\right) b_{n}\left(r_{n-1}\right)-d_{n}\left(r_{n-1}\right) a_{n}\left(r_{n-1}\right)\right)^{-1}, \quad(n=\overline{1, N}) \quad \text { and } \\
& \Lambda_{n}=\Delta_{n}\left(\begin{array}{ll}
a_{n}(r) & i b_{n}(r) \\
c_{n}(r) & i d_{n}(r)
\end{array}\right)\left(\begin{array}{ll}
-d_{n}\left(r_{n-1}\right) & b_{n}\left(r_{n-1}\right) \\
-i c_{n}\left(r_{n-1}\right) & i a_{n}\left(r_{n-1}\right)
\end{array}\right), \quad(n=\overline{1, N}) .
\end{aligned}
$$

Here

$$
\begin{aligned}
& a_{n}=-\frac{\kappa_{n}^{2}}{r} \cos \left(\kappa_{n} r\right)+\frac{\kappa_{n}}{r} \sin \left(\kappa_{n} r\right), \quad b_{n}=-\frac{\kappa_{n}}{r^{2}} \cos \left(\kappa_{n} r\right)-\frac{\kappa_{n}^{2}}{r} \sin \left(\kappa_{n} r\right), \\
& c_{n}=\frac{\kappa_{n}}{r}\left[\frac{4 \mu_{n} \kappa_{n}}{r} \cos \left(\kappa_{n} r\right)-\left(\frac{4 \mu_{n}}{r^{2}}-\rho_{n} \omega^{2}\right) \sin \left(\kappa_{n} r\right)\right], \\
& d_{n}=\frac{\kappa_{n}}{r}\left[\frac{4 \mu_{n} \kappa_{n}}{r} \sin \left(\kappa_{n} r\right)+\left(\frac{4 \mu_{n}}{r^{2}}-\rho_{n} \omega^{2}\right) \sin \left(\kappa_{n} r\right)\right], r_{n-1}<r<r_{n}, \quad(n=\overline{1, N}) .
\end{aligned}
$$

Then from the conditions of displacement and normal stress continuity at the boundaries of layers one obtains

$$
\left(\begin{array}{l}
u_{N}(r) \\
\sigma_{N}(r)
\end{array}\right)=G\left(\begin{array}{l}
u_{1}\left(r_{0}\right) \\
\sigma_{1}\left(r_{0}\right)
\end{array}\right)
$$

where $G$ is a square matrix and

$$
G=\left(\begin{array}{ll}
g_{11} & g_{12} \\
g_{21} & g_{22}
\end{array}\right) \equiv \Lambda_{n} \Lambda_{n-1} \cdots \Lambda_{1}
$$


In this case the boundary conditions (2.4) are satisfied:

$$
\sigma_{N}\left(r_{N}\right)=\sigma_{1}\left(r_{0}\right)=0
$$

From the solution (2.12), with regard for the boundary conditions (2.14), one can obtain a characteristic equation to determine frequency $\omega$ :

$$
g_{21}=0 \text {. }
$$

Its roots can be determined by Muller's method [8], while the natural frequency of elastic oscillations is taken as the initial guess.

The solutions obtained can be verified by comparing them with the calculations of V. V. Alyokhin [1], made for a sphere consisting of three elastic materials, the physical characteristics of which are presented in Table 1 . The first, second and third layers are composed of the third, second and first materials of Table 1, respectively; $r_{0}=0.7718, r_{1}=0.7901, r_{2}=0.8266, r_{N}=1.0$. Calculations lead to the square of a base natural frequency equal to 65.01 , which is equal to V. V. Alyokhin's result [1].

\section{An optimization problem}

Consider the problem of synthesis of a multilayer spherical shell from the given set of viscoelastic materials that provides maximum damping of free oscillations. The inner and outer radii of shell are fixed, $\sigma_{r}$ and $u$ play the role of phase variables.

Selecting a function $\theta(x)$ characterizing material structure, that is, presence at a given point $x$ of some material from the set, one can formulate the optimization problem as follows. Among the piecewise-constant functions $\theta(x)$ with a range of values belonging to the given finite discrete set $W$,

$$
\theta(x) \in W=\left\{\theta_{1}, \ldots, \theta_{m}\right\}
$$

where $m$ is a number of different materials, find the control $\theta(x)$ which maximizes the functional

$$
F=\operatorname{Im}[\omega(\theta)]
$$

For harmonic oscillations the system (2.1)-(2.4) can be reduced to the boundary value problem for a vector equation

$$
\vec{z}^{\prime}=A \vec{z}^{\prime} ; \quad z_{2}(0)=z_{2}(1)=0,
$$


where a new variable $x \in[0,1]$ is introduced which is related to the former one by $r=r_{0}+x\left(r_{N}-r_{0}\right)$. The prime means differentiation with respect to $x$, the vector $z=\left\{z_{1} \equiv u, z_{2} \equiv \sigma_{r}\right\}$ and the matrix $A$ is given by

$$
A=\left|\begin{array}{cc}
-2 \lambda g r & g r^{2} \\
4 \mu(3 \lambda+2 \mu) g-\rho \omega^{2}\left(r_{N}-r_{0}\right) & 4 \mu g r
\end{array}\right|,
$$

where $g=\left(r_{N}-r_{0}\right) / r^{2}(\lambda+2 \mu)$.

The square of the frequency can be expressed in phase variables (the Rayleigh method). Let us multiply the first equation of the system (3.2) by $z_{1} r^{2}$ and integrate over $x$ from 0 to 1 . Using the rule of integration by parts and the first equation of the system (3.2) one obtains

$$
\omega^{2}(\theta)=\int_{0}^{1} J_{1}(\vec{z}, \theta) d x / \int_{0}^{1} J_{2}(\vec{z}, \theta) d x
$$

where

$$
J_{1}(\vec{z}, \theta)=\left\{\frac{4 \mu(3 \lambda+2 \mu)}{r^{2}(\lambda+2 \mu)} z_{1}^{2}+\frac{1}{\lambda+2 \mu} z_{2}^{2}\right\} r^{2}, \quad J_{2}(\vec{z}, \theta)=\rho z_{1}^{2} r^{2} .
$$

To derive necessary optimality conditions let us first find variations of the functional $F_{1}=\omega^{2}(\theta)$ determined by the perturbed control

$$
\theta^{*}(x)= \begin{cases}\bar{\theta}(x), & x \in M, \bar{\theta} \in W, \text { mes } M \ll 1, \\ \theta(x), & x \notin M .\end{cases}
$$

It follows from (3.3) that

$$
\begin{aligned}
& \delta F_{1}(M, \theta)=2 \omega \delta(\omega) \\
& =\int_{M}\left[J_{1}(\vec{z}, \bar{\theta})-J_{1}(\vec{z}, \theta)-\omega^{2} J_{2}(\vec{z}, \bar{\theta})+\omega^{2} J_{2}(\vec{z}, \theta)\right] d x / \int_{0}^{1} J_{2} d x \\
& \quad+\int_{0}^{1}\left[\frac{\partial J_{1}}{\partial \vec{z}}-\omega^{2} \frac{\partial J_{2}}{\partial \vec{z}}\right] \delta \vec{z} d x / \int_{0}^{1} J_{2} d x .
\end{aligned}
$$

The last term in braces corresponds to variations of phase variables $\vec{z}$. By virtue of (3.4) it can be transformed as follows:

$$
\begin{aligned}
\int_{0}^{1} & {\left[\frac{\partial J_{1}}{\partial \vec{z}}-\omega^{2} \frac{\partial J_{2}}{\partial \vec{z}}\right] \delta \vec{z} d x } \\
& =\int_{0}^{1} z_{2} r^{2}\left(\frac{2}{\lambda+2 \mu} \delta z_{2}-\frac{4 \lambda}{r(\lambda+2 \mu)} \delta z_{1}\right) d x-2 \int_{0}^{1}\left[\frac{r^{2} z_{2}^{2}}{r_{n}-r_{0}} \delta\left(\frac{d z_{1}}{d x}\right)\right] d x .
\end{aligned}
$$


Using the first equation of the system (3.2), after a number of transformations one obtains

$$
\int_{0}^{1}\left[\frac{\partial J_{1}}{\partial \vec{z}}-\omega^{2} \frac{\partial J_{2}}{\partial \vec{z}}\right] \delta \vec{z} d x=\frac{2 r^{2} z_{2}^{2}}{r_{n}-r_{0}} \int_{M}\left[-\left(f_{1}(\bar{\theta})-f_{1}(\theta)\right)\right] d x .
$$

Here $f_{1}$ is the right-hand side of the first equation of the system (3.2).

Substituting (3.6) into (3.5) one obtains

$$
\begin{aligned}
\delta F_{1}(M, \bar{\theta})=\int_{M}\left[J_{1}(\bar{\theta})-J_{1}(\theta)-\omega^{2}\left(J_{2}(\bar{\theta})-J_{2}(\theta)\right)\right. \\
\left.\quad-\frac{2 z_{2} r^{2}}{r_{N}-r_{0}}\left(f_{1}(\bar{\theta})-f_{1}(\theta)\right)\right] d x / \int_{0}^{1} J_{2} d x .
\end{aligned}
$$

Then, varying the functional (3.1), one can write

$$
\delta F=\operatorname{Im} \delta(\omega)=\operatorname{Im}\left(\frac{\delta F_{1}}{2 \omega}\right)
$$

Bearing (3.7) in mind, the last expression takes the form

$$
\delta F=\operatorname{Im}\left\{\frac{1}{2 \omega}\left[\int_{0}^{1} J_{2} d x\right]^{-1} \int_{M}[H(\cdot, \bar{\theta})-H(\cdot, \theta)] d x\right\},
$$

where

$$
H(\cdot, \bar{\theta})=J_{1}(\cdot, \bar{\theta})-\omega^{2} J_{2}(\cdot, \bar{\theta})-\frac{2 z_{2} r^{2}}{r_{N}-r_{0}} f_{1}(\cdot, \bar{\theta})
$$

Using (3.8) as a variation of the functional (3.1), one can formulate the necessary optimality condition $\delta F(\vec{z}, \theta) \leq 0$ in the form of the maximum principle : let $\left\{\theta^{\text {opt }}\right\}$ be the optimal control in the problem (3.1), (3.2), (3.3), (3.5) having a nontrivial solution of the system (3.2). Then the Hamiltonian function (3.9) takes a minimum over the argument $\theta$ for the optimal control $\theta^{\text {opt }}$ almost at each $x \in[0,1]$, that is,

$$
H\left(\cdot, \theta^{\text {opt }}\right)=\min _{\theta \in W} H(\cdot, \theta) .
$$

In the problem considered, the Hamiltonian (3.9) does not include conjugate variables.

The existence of the problem considered has been proved in [5]. The proof is based on the introduction of new control variables which change the vector diagram of the controlled system from concave to convex. 
TABLE 2. Characteristics of viscoelastic materials and corresponding values of $\operatorname{Re} \omega$ and $\operatorname{Im} \omega$.

\begin{tabular}{|c|c|c|c|c|c|c|c|c|}
\hline No. of the material & $\rho$ & $E$ & $\nu$ & $A$ & $\alpha$ & $\beta$ & $\operatorname{Re} \omega$ & $\operatorname{Im} \omega \times 10^{3}$ \\
\hline 1 & 1 & 15 & 0.25 & 0.01 & 0.5 & 0.05 & 7.04 & 9.00 \\
\hline 2 & 2 & 40 & 0.25 & 0.01 & 0.5 & 0.05 & 8.13 & 9.70 \\
\hline 3 & 4 & 85 & 0.25 & 0.01 & 0.5 & 0.05 & 8.35 & 9.89 \\
\hline
\end{tabular}

\section{Particular examples}

The foregoing enables a computational algorithm to be formulated as follows:

(1) Using the uniform mesh of points $\left\{x_{n}\right\}$, divide the interval $[0,1]$ into a sufficiently large number of small segments of length $h=x_{n+1}-x_{n}$, simulating a set of small measure $M$.

(2) The initial guess of control $\{\theta(x)\}$ being known, determine the natural frequency by solving (2.15).

(3) Using the frequency obtained, solve the system (2.12) assuming that the values of a vector of phase variables on the segment $M_{i}$ are characterized by its value in the middle of the segment $x=x_{n}+h / 2$.

(4) Assign a new control $\{\bar{\theta}]$ on $M_{n}$ from the condition

$$
H\left[\vec{z}\left(x_{n}+h / 2\right), \bar{\theta}\right]=\min _{\theta \in W} H\left[\vec{z}\left(x_{n}+h / 2\right), \theta\right] .
$$

The process is considered to be over if the control does not change on any of the segments. When the control $\theta(x)$ takes the same value on two or several adjacent segments $M_{i}$ these segments are combined in one macrolayer. The convergence of the algorithm and its rate were carefully checked for the corresponding elastic problem [5]. Several direct calculations which we performed showed that the rate of convergence improved in the case of viscoelasticity.

Consider some typical examples. For all the variants it is required to synthesize a spherical shell with maximum damping of free oscillations.

(1) There is a set of three viscoelastic materials, characteristics of which (in a dimensionless form) are presented in Table 2. The internal and external radii of a shell are fixed: $r_{0}=0.8$ and $r_{N}=1.0$.

This variant is characterized by the fact that rheological parameters of all the materials are the same. The last two columns in Table 2 show the real and imaginary parts of the natural frequency of corresponding one-layer shells. A three-layer shell synthesized with the help of the described algorithm has the following parameters: $\operatorname{Re} \omega=8.47$ and $\operatorname{Im} \omega=9.94 \times 10^{-3}$. Its structure is presented in Figure 1(a) where the inside numbers correspond to the number of a material in Table 2. 
(a)

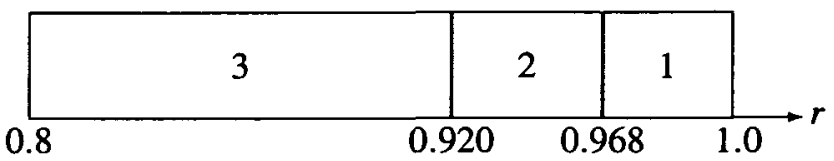

(b)

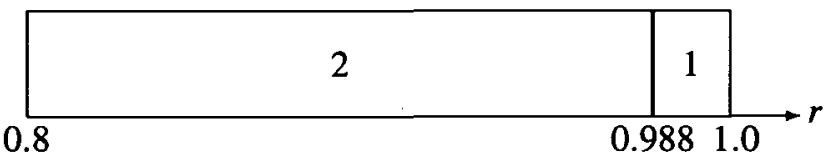

(c)

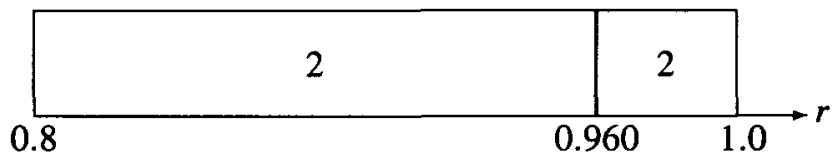

FIGURE 1. The schematic representation of the designed shells (letters a, b and c correspond to three variants in the text).

TABLE 3. Relaxation parameter of materials and corresponding values of $\operatorname{Re} \omega$ and $\operatorname{Im} \omega$.

\begin{tabular}{|c|c|c|c|}
\hline No. of the material & $\alpha$ & $\operatorname{Re} \omega$ & $\operatorname{Im} \omega \times 10^{3}$ \\
\hline 1 & 0.10 & 6.89 & 25.7 \\
\hline 2 & 0.12 & 8.00 & 27.0 \\
\hline 3 & 0.15 & 8.28 & 25.3 \\
\hline
\end{tabular}

(2) In this example all the characteristics of materials are the same as in Table 2, except for the parameter $\alpha$. Its values and corresponding real and imaginary parts of complex frequency are shown in Table 3.

Maximum damping of natural frequency is provided by a two-layer spherical shell with $\operatorname{Re} \omega=8.0, \operatorname{Im} \omega=27.2 \times 10^{-3}$ presented in Figure 1(b).

(3) Three materials are given, the parameters of which are presented in Table 4. The synthesized shell obtained is a two-layer one (Figure 1(c)) with the following characteristics: $\operatorname{Re} \omega=5.96$ and $\operatorname{Im} \omega=7.6 \times 10^{-3}$.

\section{Conclusion}

The analysis of the results leads firstly to a positive answer to the question formulated at the beginning of the paper: damping capability does increase due to peculiarities of wave propagation through the boundaries of different viscoelastic ma- 
TABLE 4. Characteristics of materials and corresponding values of $\operatorname{Re} \omega$ and $\operatorname{Im} \omega$.

\begin{tabular}{|c|c|c|c|c|c|c|c|c|}
\hline No. of the material & $\rho$ & $E$ & $\nu$ & $A$ & $\alpha$ & $\beta$ & $\operatorname{Re} \omega$ & $\operatorname{Im} \omega \times 10^{3}$ \\
\hline 1 & 1 & 15 & 0.25 & 0.01 & 0.8 & 0.05 & 7.05 & 4.4 \\
\hline 2 & 4 & 48 & 0.20 & 0.01 & 0.5 & 0.05 & 5.77 & 7.3 \\
\hline 3 & 8 & 42 & 0.15 & 0.01 & 0.5 & 0.05 & 5.50 & 6.5 \\
\hline
\end{tabular}

terials. However it must be mentioned that in all the cases considered the increase of damping is not large in comparison to that in a one-layer shell of "the best" material (maximum value of $\operatorname{Im} \omega$ in Tables 2-4). Secondly, in all the layered shells the first inside layer always consists of this "best" material, its thickness as a rule being much larger than that of the other layers. This can be seen in the second example in particular (Figure 1(b)).

\section{References}

[1] V. V. Alyokhin, "Optimal design of inhomogeneous-elastic and layered bodies", Ph. D. Thesis, Cand. Sci. (Phys.-Math.), (Novosibirsk, 1986).

[2] G. D. Babe and E. L. Gusev, Mathematical methods of optimization of interference filters (Nauka, Novosibirsk, 1987).

[3] A. M. Filatov, Asymptotic methods in the theory of differential and integral equations (Fan, Tashkent, 1974).

[4] E. L. Gusev, Mathematical methods of synthesis of layered structures (Nauka, Novosibirsk, 1993).

[5] M. A. Kanibolotsky and Yu. S. Urzhumtsev, Optimal design of layered structures (Nauka, Novosibirsk, 1989).

[6] M. A. Koltunov, V. P. Maiboroda and A. S. Kravchuk, Applied mechanics of a strained solid body (Higher School, Moscow, 1983).

[7] M. A. Koltunov, Creep and relaxation (Higher School, Moscow, 1976).

[8] G. Korn and T. Korn, Mathematical handbook for scientists and engineers (McGraw-Hill, 1968).

[9] V. P. Maiboroda and I. E. Troyanovsky, "Free oscillations of inhomogeneous viscoelastic bodies", USSR Ac. Sci., J. of Mechanics of Solids 2 (1983), 117-123. 